\title{
OPTIMIZATION OF THE NORM OF THE LAGRANGE INTERPOLATION OPERATOR
}

\author{
BY T. A. KILGORE
}

\section{Communicated by Chandler Davis, December 2, 1976}

On an interval $[a, b]$, we may place points $t_{0}, \ldots, t_{n}$ such that $a=t_{0}<$ $t_{1}<\cdots<t_{n}=b$. Using these points, called nodes, we may construct unique polynomials $y_{0}, \ldots, y_{n}$ of degree $n$, such that, for $1 \leqslant i, j \leqslant n, y_{i}\left(t_{i}\right)=1$ and $y_{i}\left(t_{j}\right)=0$ for $j \neq i$. The Lagrange interpolating projection on the nodes $t_{0}, \ldots, t_{n}$ is the operator which takes any function $f$ continuous on $[a, b]$ to the polynomial $\sum_{i=0}^{n} f\left(t_{i}\right) y_{i}$. It is easily seen that this projection is bounded for any degree $n$, for any interval $[a, b]$, and for any set of nodes in $[a, b]$. The norm is easily shown to be the sup norm of $\Lambda=\sum_{i=0}^{n}\left|y_{i}\right|$, called the Lebesgue function of the projection, and thus the norm depends exclusively on the placement of $t_{1}, \ldots, t_{n-1}$. It is irrelevant, in attempting to minimize the norm, to move $t_{0}$ or $t_{n}$. Of the function $\Lambda$, it is true that $\Lambda\left(t_{i}\right)=1$ for $0 \leqslant i \leqslant n$, while if $n \geqslant 2$ and if $t$ is not a node, then $\Lambda(t)>1$. Let $\lambda_{1}, \ldots, \lambda_{n}$ be the values given by

$$
\lambda_{i}=\sup _{t \in\left[t_{i-1}, t_{i}\right]} \Lambda(t) \text { for } 1 \leqslant i \leqslant n .
$$

Then $\|\Lambda\|=\max _{1 \leqslant i \leqslant n} \lambda_{i}$.

It was conjectured by Serge Bernstein in 1932 that the norm of the interpolating projection is minimized when the nodes are so placed that $\lambda_{1}=\cdots=\lambda_{n}$, a conjecture rendered plausible, but by no means demonstrated, by the rather obvious fact that

$$
\frac{\partial \lambda_{i}}{\partial t_{i}}>0>\frac{\partial \lambda_{i+1}}{\partial t_{i}}, \text { for } 1 \leqslant i \leqslant n-1,
$$

and by the fact that moving any node into close proximity with one of its neighbors increases $\|\Lambda\|$ without bound. This communication will give the following theorem and an outline of its proof in a series of lemmas.

THEOREM. For any $n \geqslant 2$, if the norm of the Lagrange interpolation operator on an interval $[a, b]$ with nodes $a=t_{0}<t,<\cdots<t_{n}=b$ is to be minimized, then it is necessary that the local maximum values $\lambda_{1}, \ldots, \lambda_{n}$ of the Lebesgue function be equalized.

The proof of this theorem depends on the fact that $\left(\lambda_{1}, \ldots, \lambda_{n}\right)$ is a dif- 
ferentiable function of $t_{1}, \ldots, t_{n-1}$. For $1 \leqslant i \leqslant n$ we denote by $X_{i}$ the polynomial which agrees with $\Lambda$ on $\left(t_{i-1}, t_{i}\right)$. We denote by $\tau_{i}$ the (unique) point in $\left(t_{i-1}, t_{i}\right)$ at which $X_{i}\left(\tau_{i}\right)=\lambda_{i}$. It is then established that, for $1 \leqslant i \leqslant n$ and $1 \leqslant j \leqslant n-1, \partial \lambda_{i} / \partial t_{j}=-y_{j}\left(\tau_{i}\right) X_{i}^{\prime}\left(t_{j}\right)$. Our theorem follows if we can show that, for any position of nodes, every $n-1 \times n-1$ submatrix of $\left(\partial \lambda_{i} / \partial t_{j}\right)_{i j}$ is nonsingular $(1 \leqslant i \leqslant n$ and $1 \leqslant j \leqslant n-1)$. Using the above expression for $\partial \lambda_{i} / \partial t_{j}$, we may with artful cancellations reach the equivalent matrix $\left(q_{i}\left(t_{j}\right)\right)_{i j}$, for $1 \leqslant$ $i \leqslant n, 1 \leqslant j \leqslant n-1$, where $q_{i}(t)=X_{i}^{\prime}(t) /\left(t-\tau_{i}\right)$ is a polynomial of degree $n-2$ or less. ${ }^{1}$ Thus each $n-1 \times n-1$ submatrix of $\left(q_{i}\left(t_{j}\right)\right)$ is nonsingular if and only if any $n-1$ of $q_{1}, \ldots, q_{n}$ form a basis for the space of polynomials of degree $n-2$ or less. In a succession of lemmas, we show that any $n-1$ of $q_{1}, \ldots, q_{n}$ are indeed a basis. Here, for reasons of conserving space, the proofs are not given in full.

Lemma 1. The polynomials $X_{1}$ and $X_{n}$ have their full complement of $n$ roots on $[a, b]$, and $X_{1}^{\prime}$ and $X_{n}^{\prime}$ have their full complement of $n-1$ roots on $[a, b]$. For each $i, 2 \leqslant i \leqslant n-1$, the polynomial $X_{i}$ has exactly $n-1$ roots on $[a, b]$, and $X_{i}^{\prime}$ has at least $n-2$ roots on $[a, b]$. Each root of each of the above polynomials has multiplicity one, and each root of $X_{i}^{\prime}, 1 \leqslant i \leqslant n$, is a local extremum of $X_{i}$.

Proof of Lemma 1. An obvious counting argument suffices.

Lemma 2. For $2 \leqslant i \leqslant n, X_{i-1}^{\prime}$ and $X_{i}^{\prime}$ have no common root, nor do $X_{1}^{\prime}$ and $X_{n}^{\prime}$.

Proof. One uses $X_{i-1}+X_{i}=2 y_{i-1}$ and an analogous identity for $X_{1}$ and $X_{n}$. If for example $X_{i-1}^{\prime}$ and $X_{i}^{\prime}$ have a common root, then so does $X_{i-1}^{\prime}+$ $\alpha y_{i-1}^{\prime}$, for any real $\alpha$. One then investigates the number of roots of $X_{i-1}+$ $\alpha y_{i-1}$, when $\alpha$ is chosen so that $\left(X_{i-1}+\alpha y_{i-1}\right)(r)=0$, at an assumed common root $r$ of $X_{i-1}^{\prime}$ and $X_{i}^{\prime}$.

Lemma 3. All roots of $X_{1}^{\prime}$ and $X_{n}^{\prime}$ lie on $\left[\tau_{1}, \tau_{n}\right]$.

Proof. Since $X_{1}^{\prime}$ and $X_{n}^{\prime}$ have no common root, one can investigate convenient extreme configurations of $t_{1}, \ldots, t_{n-1}$.

LEMMA 4. The roots of $X_{1}^{\prime}$ and $X_{n}^{\prime}$ alternate as we pass from $\tau_{1}$ to $\tau_{n}$.

Proof. Similar to Lemma 3.

LEMMA 5. Between $\tau_{1}$ and the first root of $X_{n-1}^{\prime}$ appearing on $\left[\tau_{1}, \tau_{n}\right]$, there is a root of $X_{n}^{\prime}$. The symmetric statement about $\tau_{n}, X_{2}^{\prime}$, and $X_{1}^{\prime}$ also holds.

Proof. Similar to Lemma 3.

Lemma 6. On the interval $\left[\tau_{1}, \tau_{n}\right]$ the roots of $X_{1}^{\prime}, \ldots, X_{n}^{\prime}$ lie in the

1 Private communication from Dietrich Braess. 
pattern $\hat{1}, n, n-1, \ldots, 3, \hat{2}, 1, n, \ldots, 4, \hat{3}, 2,1, n, \ldots, 1, n, \widehat{n-1}, n-2$, $\ldots, 1, \hat{n}$, when a number $i$ denotes a root of $X_{i}, 1 \leqslant i \leqslant n$, and where $\hat{i}$ denotes the point $\tau_{i}, 1 \leqslant i \leqslant n$.

Proof. This proof uses the method of Lemmas 3, 4, 5, and the results of all previous lemmas, and the method of induction.

Corollary of Lemma 6. The roots of $q_{1}, \ldots, q_{n}$ lie in the same locations as those of $X_{1}^{\prime}, \ldots, X_{n}^{\prime}$, save that $\tau_{1}, \ldots, \tau_{n}$ are removed from the list.

Proof. Clear.

LEMMA 7. Without doing harm, we may assume that $q_{i}\left(\tau_{1}\right)>0$ for $1 \leqslant$ $i \leqslant n$. Under this convention, we have $\operatorname{sgn} q_{i}\left(\tau_{i}\right)=\operatorname{sgn} q_{1}\left(\tau_{i}\right)$ and $\operatorname{sgn} q_{j}\left(\tau_{i}\right)=$ $-\operatorname{sgn} q_{1}\left(\tau_{i}\right)$ for $j \neq i$, where $2 \leqslant i, j \leqslant n$.

Proof. Follows easily from Lemma 6 and its Corollary.

Lemma 8. The set $\left\{q_{1}, \ldots, q_{n}\right\} \sim\left\{q_{k}\right\}$ is linearly independent for any choice of $k, 1 \leqslant k \leqslant n$.

Proof. We assume the existence of a nontrivial linear combination $\alpha_{1} q_{1}+$ $\cdots+\alpha_{n} q_{n}=0$, in which, for some $k, 2 \leqslant k \leqslant n$, we have assumed $\alpha_{k}=0$, and also $\alpha_{1} \geqslant 0$. The sets $N=\left\{j \mid \alpha_{j}<0\right.$ and $\left.2 \leqslant j \leqslant n\right\}$ and $P=\left\{j \mid \alpha_{j}>0\right.$ and $2 \leqslant$ $j \leqslant n\}$ are shown nonempty, and the polynomial $N=\alpha_{1} q_{1}+\Sigma_{j \in N} \alpha_{j} q_{j}$ is shown to alternate sign at successive points $\tau_{i}, 1 \leqslant i \leqslant n$, thus having at least $n-1$ roots, while having degree no more than $n-2$. The contradiction implies the lemma.

Our theorem now follows from Lemma 8, in light of previous discussion.

ADDED MAY 18, 1977. In the paper which gives details of the above theorem, I complete the proof of Bernstein's conjecture by proving that there is a unique choice of nodes which equalizes the $\lambda_{i}$. I have been informed by $\mathrm{C}$. de Boor and A. Pinkus that they too, using the results of the present note, have completed the proof of Bernstein's conjecture.

DEPARTMENT OF MATHEMATICS, KING ABDULAZIZ UNIVERSITY, MECCA, SAUDI ARABIA 